\title{
Une délétion du proto-oncogène c-kit dans une maladie cutanée humaine, le piébaldisme
}

En décembre 1990, $\mathrm{m} / \mathrm{s}$ publiait une nouvelle $\left(n^{\circ} 10\right.$, vol. 6, p. 1016) faisant part d'une découverte importante en génćtique de la souris: celle du système W; c-kit/Steel. En bref, ce système code pour deux protéines génétiquement distinctes mais physiologiquement associées : un premir gène code pour un proto-oncogène, $c-k i t$, qui est un récepteur membranaire doté d'une activité tyrosine kinase, et qui est muté chez les souris porteuses des allèles $\mathrm{W}$; le second code pour un ligand de la protéine c-Kit, et dont le gène est altéré par les mutations dites Steel ou SI. Dans les deux cas les symptômes sont les mêmes : à l'état homozygote, deux ou trois systèmes sont atteints ; la peau, entièrement dépigmentée ; l'hématopoïèse, avec une anémie macrocytaire profonde; et, pour celles qui survivent, une stérilité totale. Les hétérozygotes, en revanche, présentent uniquement des troubles cutanés, sous forme d'une tache blanche frontale et de zones de dépigmentation localisées. Il était tentant de rechercher chez l'homme des maladies comparables. Parmi elles, le piébaldisme (de piebald, pie), une forme d'albinisme partiel, paraît très semblable à la forme tachetée dominante de la souris : hérédité autosomique dominante, mèche blanche frontale, zones limitées de dépigmentation (leucoderme), sans localisations extracutanées. Une équipe unissant des chercheurs de Dallas (TX, USA) et de Genelabs, Redwood City (CA, USA), a entrepris l'étude du gène $c$-kit dans l'ADN de sept sujets non apparentés atteints de pićbaldisme. Le gène $c$-kit a pu être localisć sur la partie proximale du bras long du chromosome 4 , en 4qll-12. Chez un des malades, on a pu démontrer l'existence d'une délétion incluant le locus c-kit par deux méthodes:: l'hybridation classique de l'ADN avec des sondes du chromo- some 4 , et la méthode, qui se développe très rapidement, dite FISH (fluorescence in situ hybridization), qui, avec une sonde de $c$-kit, révèle deux signaux sur les chromosomes normaux et un seul en cas de délétion [1].

Le locus du piébaldisme avait déjà été auparavant assigné à la région proximale de 4q sur la foi de délétions cytogénétiquement visibles, mais des anomalies associées empêchaient une localisation précise [2]. Dans le cas décrit dans (1), indétectable au microscope, un autre locus est cependant délété, celui du PDGFR $\alpha$ (platelet denived growth factor receptor $\alpha$ ). Ce dernier gène joue probablement un rôle important dans le développement, mais, d'après les tableaux observés lors d'autres délétions, ne semble pas pouvoir être responsable du piébaldisme.

Ce travail soulève nombre de questions intéressantes. Sur le résultat d'abord : si l'observation paraît probante, elle est unique à ce jour. Comme le font remarquer les auteurs eux-mêmes, les mutations ponctuelles sont en général plus fréquentes que les délétions ; on attend donc avec intérêt l'analyse des gènes des six autres malades. Il est certain qu'une lésion du c-kit seul, surtout si on peut en montrer les conséquences fonctionnelles, entraînerait la conviction. Sur le plan clinique, on peut se demander pourquoi chez des hétérozygotes, avec une mutation présente en principe dans toutes les cellules, on observe une alternance de zones normales et de zones complètement dépigmentées. On ne peut, à l'heure actuelle, émettre que des hypothèses non démontrées, mais deux problèmes paraissent particulièrement passionnants.

Le premier a trait à la comparaison des effets d'une même anomalie dans deux espèces, ici homme et souris. Chez les hétérozygotes existe une res- semblance frappante, visible nettement sur les photographies : même symptômes, et stricte limitation au système cutané ; aucun trouble hématologique ni de la fertilité n'a été décrit. Mais, dans une famille de malades avec troubles cutanés étendus [3] on a observé un retard mental et des troubles cérébelleux. Plus encore, on connaît l'existence d'un enfant homozygote [4], dont les deux parents ont les signes du piébaldisme; il présente, non seulement une absence totale de pigmentation pileuse et cutanée, mais également un retard mental important. Les formes sévères chez l'homme paraissent donc affecter le système nerveux et non l'hématopoïèse, alors que c'est l'inverse chez la souris. On a fait toutefois remarquer que, même chez la souris, le niveau de l'ARNm de c-kit est le plus élevé dans le cerveau, surtout l'hippocampe et le cervelet [5].

On est, de plus, obligé d'être prudent dans l'interprétation d'un cas homozygote chez l'homme, tant que la nature de la mutation n'a pas été élucidée : en cas de délétion, d'autres gènes pourraient être en cause.

Le deuxième problème est celui de savoir si l'altération de plus d'un gène ne pourrait aboutir à un même tableau clinique. Si, en effet, nous retournons à la génétique de la souris, nous voyons que des mutations dans le ligand de c-Kit produit chez cet animal les allèles SI, dont les effets sont les mêmes que ceux des mutations de $c$-Kit, c'est-à-dire l'apparition du phénotype tacheté. Il serait donc parfaitement possible que chez certains malades présentant les symptômes du piébaldisme, ce soit le ligand, et non cKit lui-même, qui soit en cause. Puisque des sondes pour le gène du ligand existent, du moins chez la souris où il a été localisé sur le chromosome 10, cette hypothèse ne saurait tarder à être vérifiée. 
On peut enfin remarquer que les oncogènes sont de moins en moins confinés au domaine de la cancérologie. Après avoir investi la cardiologie $(\mathrm{m} / \mathrm{s}$ $n^{\circ} 6$, vol. 7, p. 640), ils abordent maintenant celui de la dermatologie.

J.C. D.

1. Fleischman RA, Saltman DL, Stasny V, Zneimer S. Deletion of the $c$-kit protooncongene in the human developmental defect piebald trait. Proc Nall Acad Sci USA 1991; 88 : 10885-9. 2. Hoo JJ, Haslam RHA, van Orman C. Tentative assignment of piebald trait gene to chromosome 4q12. Hum Genet 1986; 73 : 230-1. 3. Telfer MA, Sugar M, Jaeger EA, Mulcahy J. Dominant piebald trait (with forelock and leukoderma) with neurological impairment. $A m$ Med Genet 1971 ; 23 : 383-9.

4. Hultén MA, Honeyman MM, Mayne AJ,

Tarlow MJ. Homozygosity in piebald trait. $J$ Med Genet 1987; 24 : 568-71.

5. Orr-Urtreger A, Avivi A, Zimmer Y, Givol D, Yarden Y, Lonai P. Developmental expression of c-kit, a protooncogene encoded by the $\mathrm{W}$ locus. Development $1990 ; 109$ : 911-23.

\section{ERRATUM}

Nous republions ici la figure 1 du lexique embryologique $\left(\mathrm{m} / \mathrm{s} n^{\circ} 10\right.$, vol. 7 , décembre 91, p. 1081), dans sa version exacte.

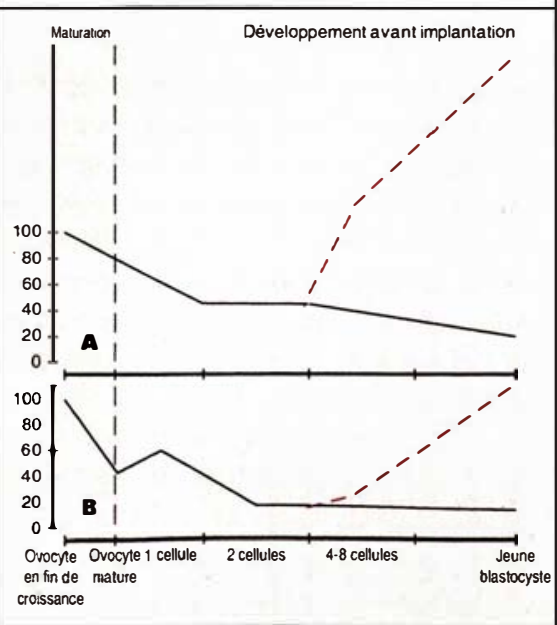

